\title{
Re-audit: transcervical resection of the endometrium for heavy menstrual bleeding and the treatment of post-resection uterine stenosis and haematometra by cervical dilatation and the insertion of a levonorgestrel-releasing intra-uterine system
}

\author{
F. M. S. Basama • H. Muppala • T. C. M. Inglis \\ Received: 18 June 2006 / Accepted: 7 August 2006 / Published online: 25 October 2006 \\ (C) Springer-Verlag 2006
}

\begin{abstract}
The audit objectives: To assess the outcome of the treatment of: (1) heavy menstrual bleeding by transcervical resection of the endometrium, (2) post-resection uterine stenosis and haematometra by cervical dilatation and levonorgestrel-releasing intra uterine system. The audit design: A retrospective re-audit. The setting: A district general hospital in the North West region of England, U.K.

The patients: Forty-seven women with heavy menstrual bleeding that required treatment with transcervical resection of the endometrium. The interventions: Transcervical resection of the endometrium, cervical dilatation and the insertion of levonorgestrel-releasing intra uterine system.

The main outcome measures: Clinical improvement and the need for further surgery, including hysterectomy. The results: Transcervical resection of the endometrium improved the symptoms of $80.9 \%$ of the women. The problem of six out of seven women with post-resection uterine stenosis and haematometra was resolved by cervical dilatation and the insertion of levonorgestrel-releasing intra-uterine system. The conclusion: Transcervical resection of the endometrium for heavy menstrual loss is a safe and effective procedure. Cervical dilatation and the insertion of levonorgestrel-releasing intra-uterine system saved some women with post-resection uterine stenosis and haematometra from having further surgery. The two interventions combined saved $93.6 \%$ of the women from having hysterectomies.
\end{abstract}

F. M. S. Basama $(\bowtie) \cdot H$. Muppala $\cdot$ T. C. M. Inglis Obstetrics and Gynaecology, Burnley General Hospital, Casterton Avenue,

Burnley, Lancashire BB10 2PQ, UK

e-mail: fbasama@hotmail.com
Keywords Heavy menstrual bleeding - Uterine stenosis . Haematometra - Transcervical resection of the endometrium . Levonorgestrel-releasing intra uterine system

\section{Introduction}

Heavy menstrual bleeding constitutes a major health problem and poses a large burden on the resources of the National Health Service. Approximately one in 20 women aged between 30 and 49 years consult their general practitioners each year with menorrhagia, which accounts for $20 \%$ of all referrals to gynaecologists and $60 \%$ of these referrals will end with hysterectomy within 5 years [1]. It has been estimated that one in five women in the UK will have hysterectomy before the age of 60 and in at least $50 \%$ of those who undergo hysterectomies, menorrhagia was the presenting symptom and approximately $50 \%$ of all women who had hysterectomies for menorrhagia had a normal uterus removed [1].

Various medical treatments and less-invasive surgical techniques were introduced in the last three decades in an attempt to reduce the recourse to hysterectomy and its associated cost and morbidity. Trans-cervical resection of the endometrium (TCRE) was the most widely used firstgeneration endometrial ablative technique. The technique is safe, effective and women find it satisfying and acceptable [2-4].

Approximately $3 \%$ of the women are likely to develop haematometra after TCRE [5]. Hormonal treatment for the residual endometrium has been reported before [6, 14]; however, we are not aware of any reports of the insertion of a levonorgestrel-releasing intra-uterine system (LNG IUS) to treat post-resection uterine stenosis and haematometra. 


\section{Previous audit}

The first audit was retrospective and covered the period between 1993 and 1997 and included 171 women who underwent TCRE. There were nine immediate postoperative complications; eight women had infection (endometritis) and one woman developed haematometra. In their first postoperative visit, the majority of the women (90\%) were satisfied with the outcome and reported either amenorrhoea or lighter periods; however, at 6-48 months follow-up 69 (40\%) of the women presented with either no improvement or deterioration of their symptoms and 44 (25.7\%) of them underwent further surgery; seven women had dilatation and curettage, 20 women had cervical dilatation and the insertion of a Copper $\mathrm{T}$ intrauterine contraceptive device, one woman had a repeat TCRE and 16 women eventually underwent hysterectomies. Following the above audit the practice was changed:

- All the women had their cervices dilated to Hegar number 8 prior to the procedure and to Hegar number 12 at the end of the procedure in order to reduce the incidence of uterine stenosis

- All women with post-resection haematometra had cervical dilatation and the insertion of LNG IUS

\section{The objectives of the re-audit}

The objectives of this re-audit were to assess the outcome of:

- TCRE for heavy menstrual periods

- Cervical dilatation and insertion of a LNG IUS for post-resection uterine stenosis and haematometra.

\section{The design}

A retrospective re-audit. The data was collected from women's hospital records using a designed proforma.

\section{Timescale}

The audit covers a period of 2 years (01/08/2003 to $31 / 08 /$ 2005).

\section{The setting}

The gynaecological department of Burnley Hospital; a district general hospital in the North West region of England, UK.

\section{The patients}

The study population was 47 women who presented with menorrhagia that required treatment with TCRE.

\section{The interventions}

- All the women had a preoperative trans-vaginal scan (TVS)

- Hysteroscopy and/or endometrial biopsy were performed in all perimenopausal women presenting with intermenstrual bleeding

- All the women received danazol $200 \mathrm{mg}$ twice daily for 4-8 weeks preoperatively

- All the women had TCRE performed under general anaesthesia

- Routinely, the cervix was dilated to Hegar number 8 prior to the procedure and to Hegar number 12 at the end of the procedure

- TCRE was performed by Storz rigid, 14-gauge resectoscope, using an angled-cutting, 7-mm loop and haemostasis was achieved with roller ball coagulation. The fluid medium was Purisol with gravity lead

- All the women received postoperative antibiotic (coamoxiclav) prophylaxis for 5 days

- All symptomatic women who developed post-resection uterine stenosis and haematometra, as diagnosed by TVS, had cervical dilatation and the insertion of LNG IUS.

\section{The main outcome measures}

- Clinical improvement

- The need for further surgery, including hysterectomy.

\section{The results}

Forty-seven women were included in the audit. Their ages ranged between 29 and 54 years and 45 of them were aged between 31 and 50 years. All the women were parous; four were para 1, 23 were para 2 and 16 were para $3-5$. All the women had heavy menstrual bleeding. The periods were regular in 16 and irregular in 31. Most women (40) had menstrual cycles longer than 21 days and were bleeding for more than 5 days per cycle. The duration of symptoms ranged between one and more than 5 years. The uterine size was normal in six women and was bulky or enlarged in the remainder; nine women had small intramural or submucous 
fibroids. The endometrium appeared to be well-suppressed on hysteroscopy in 32 women, poorly-suppressed in 12 and was not documented in three cases.

Two women bled heavily during the procedure. The bleeding was controlled by the insertion of a $30-\mathrm{mm}$ balloon catheter into the uterine cavity for $24 \mathrm{~h}$. One woman had cervical lacerations that required suturing.

In most women, the post-operative pain was manageable by simple analgesia, eight women required single dosage of morphine. Most women (43) were discharged home the following day; however, three women stayed for 2 days and one woman stayed for 3 days.

The histology of the resected endometrium was reported as; secretory in six specimens, proliferative phase in 18 , inactive in 21, showing foci of adenomyosis in 36 and none of the samples showed neoplastic features.

The long-term follow-up ranged between 7 and 30 months, the majority of women were followed for 2 years. At the 3- to 6-months follow-up, four women complained of pain, no change in symptoms, or increased bleeding. The four women, had haematometra diagnosed on TVS and all had cervical dilatation and the insertion of LNG IUS; three improved and the symptoms of one woman continued and she opted for total abdominal hysterectomy and bilateral salpingo-oophrectomy (TAH and BSO). At the 6- to 12-months follow-up, one woman complained of pelvic pain, had haematometra diagnosed on TVS and had a LNG IUS inserted, and was discharged later. The symptoms of two women showed no improvement or deterioration and both underwent TAH and BSO. At $>1$ year, two patients complained of pelvic pain and had haematometra diagnosed on TVS and both underwent cervical dilatation and the insertion of LNG IUS; both improved and were discharged later.

In summary, the outcome of TCRE was unsatisfactory in only nine women out of 47 ;

- Seven women presented at subsequent follow-ups with pelvic pain and were diagnosed to have uterine stenosis and haematometra and all of them had cervical dilatation and the insertion of LNG IUS; six were eventually discharged and one woman had TAH and BSO for irregular bleeding and pain

- The eighth woman had no improvement in her bleeding patterns and underwent $\mathrm{TAH}$ and $\mathrm{BSO}$

- The symptoms of the ninth woman worsened and she opted for TAH and BSO

\section{Discussion}

The symptoms of 38 women (80.9\%) improved after TCRE alone. The problems of further six women out of seven $(85.7 \%)$ with post-resection uterine stenosis and haematometra were cured by cervical dilatation and the insertion of LNG IUS. TCRE, cervical dilatation and the insertion of LNG IUS, combined, resolved the menstrual problems of $93.6 \%$ of the women.

The result of this re-audit and the previous audit showed that TCRE is a safe and effective in the treatment of heavy menstrual bleeding. There were some complications encountered; however, they were all minor and all responded to simple measures. Although TCRE for heavy menstrual bleeding may not avoid hysterectomy in all women, nevertheless the procedure remained conservative with low morbidity and provided a high chance of avoiding the need of a more radical surgery.

Hunter and McClelland [7] and Pooley et al. [8] reckoned that TCRE requires skilled surgeons. Undeniably, this is applicable not only to TCRE, but rather to any surgical procedure, which should only be performed by appropriately-trained personnel. The skills of TCRE should not be wasted just for the sake of the newer endometrial ablation techniques, whose effectiveness are yet to be proved. Cooper et al. [3] found that both microwave endometrial ablation (MEA) and TCRE achieved significant and comparable improvements in menstrual symptoms and quality of life; however, the authors thought that MEA was superior to TCRE. Undoubtedly, the newer ablative techniques are easier to perform, but not necessarily the most effective; recent evidence suggests that the outcome profile (in term of success or complications) for both TCRE and the newer endometrial ablative techniques is not different [9]. O'Connor et al. [10] found that TCRE provides the benefits of shorter operating time, fewer complications and faster rates of recovery, and they concluded that the technique is an acceptable alternative to hysterectomy in the treatment of menorrhagia. Pooley et al. [8] regarded TCRE as an effective conservative technique, with a low morbidity and a high chance of avoiding the need for major surgery, especially when performed in women over 45 years of age and by an experienced surgeon. A more recent comprehensive work by Boe Engelsen et al. [2] revealed high patient satisfaction with TCRE and that the procedure resolved the problem of heavy periods in $83.4 \%$ of the women treated; thus avoiding major surgery. The authors also found that the long-term results did not correlate to surgeon's experience.

TCRE is safe and effective; however, its efficiency can potentially be reduced by the development of inflammation, incomplete excision and regeneration of the endometrium, or adenomyosis $[11,12]$. The repairing patterns after TCRE involve granulation and scar formation, and this may explain the development of post-TCRE adhesions, cavity obliteration and haematometra. 
The levonorgestrel-releasing intra-uterine system is an effective treatment for heavy menstrual bleeding [13]. In a small study, Maia et al. [14] showed that the insertion of LNG IUS immediately after TCRE is an effective treatment for menorrhagia caused by adenomyosis, with very few adverse effects. In this re-audit, the LNG IUS was inserted some months after TCRE and did resolve the problem for six out of seven women with post-resection uterine stenosis and haematometra.

Finally, a case may be made for endometrial ablation techniques against hysterectomy, but it is ironic that each woman's case should be assessed on its own merits and it has to be realised that the different options for surgery for dysfunctional uterine bleeding are not interchangeable and they represent different patterns of care [15].

\section{Conclusion}

Transcervical resection of the endometrium is a safe and effective procedure and should continue to be offered to women with menorrhagia and who are suitable for ablative techniques. This audit showed good results with minimal complications. Cervical dilatation and the insertion of LNG IUS should be considered in women who develop postresection uterine stenosis, haematometra and dysmenorrhoea. However, these results should be viewed with caution, as the numbers are too small to give firm conclusions.

Acknowledgement We would like to thank Miss Caroline Kippax in the clinical audit department, Burnley General Hospital.

\section{References}

1. RCOG (1999) National Evidence-Based Clinical Guidelines. The management of menorrhagia in secondary care. Guideline number 5. RCOG Press
2. Boe Engelsen I, Woie K, Hordnes K (2006) Transcervical endometrial resection: long-term results of 390 procedures. Acta Obstet Gynecol Scand 85(1):82-87

3. Cooper KG, Bain C, Lawrie L, Parkin DE (2005) A randomised comparison of microwave endometrial ablation with transcervical resection of the endometrium; follow up at a minimum five years. BJOG 112(4):470-475

4. Sriemevan A, Mooney P, Boto T (2000) Transcervical resection of endometrium: patient satisfaction survey at a district general hospital. J Obstet Gynaecol 20(5):517-519

5. Mints M, Radestad A, Rylander E (1998) Follow up of hysteroscopic surgery for menorrhagia. Acta Obstet Gynecol Scand 77(4):435-438

6. Jacobs SA, Blumenthal NJ (1995) Endometrial resection follow up: late onset of pain and the effect of depot medroxyprogesterone acetate. BJOG 102(7):587 Jul

7. Hunter DC, McClelland HR (1998) Trans-Cervical resection of the endometrium; the first four years' experience at the Belfast City Hospital. Ulster Med J 67(1):29-32

8. Pooley AS, Ewen SP, Sutton CJ (1998) Does transcervical resection of the endometrium for menorrhagia really avoid hysterectomy? Life table analysis of a large series. J Am Assoc Gynecol Laparosc 5(3):229-235

9. Lethaby A, Hickey M, Garry R (2006) Endometrial resection techniques for heavy menstrual bleeding. The Cochrane Database of Systematic Reviews, issue 2

10. O'Connor H, Broadbent JA, Magos AL, McPherson K (1997) Medical Research Council randomised trial of endometrial resection versus hysterectomy in management of menorrhagia. Lancet 349(9056):897-901

11. Duan H, Liang YJ, Li L, Yang Y, Xia EL (2003) Research on repairing patterns and factors causing subsequent surgery after transcervical resection of endometrium. Zhonghua $\mathrm{Fu}$ Chan $\mathrm{Ke} \mathrm{Za}$ Zhi 38(12):741-744

12. Turnbull LW, Jumaa A, Bowsley SJ, Dhawan S, Horsman A, Killick SR (1997) Magnetic resonance imaging of the uterus after endometrial resection. BJOG 104(8):934-938

13. Lethaby AE, Cooke I, Rees M (2006) Progesterone or progestogen-releasing intrauterine systems for heavy menstrual bleeding. The Cochrane Database of Systematic Reviews, issue 2

14. Maia H Jr, Maltez A, Coelho G, Athayde C, Coutinho EM (2003) Insertion of Mirena after endometrial resection in patients with adenomyosis. J Am Assoc Gynecol Laparosc 10(4):512-516

15. Clarke A, Judge A, Herbert A, McPherson K, Bridgman S, Maresh M, Overton C, Altman D (2005) Readmission to hospital 5 years after hysterectomy or endometrial resection in a national cohort study. Qual Saf Health Care 14:41-47 